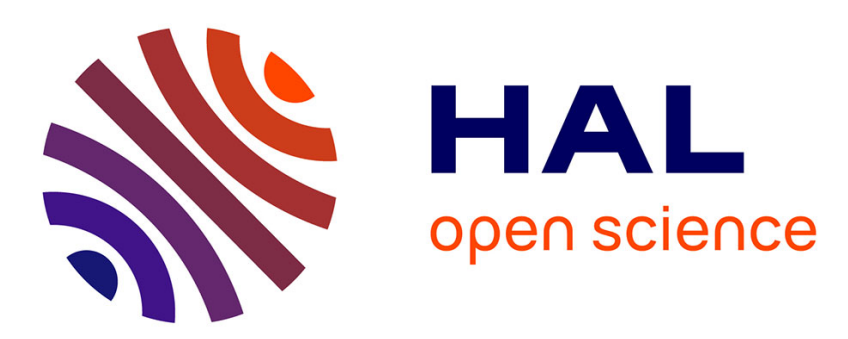

\title{
Towards a Rational Morphology Control of Frozen Copolymer Aggregates
}

Laurence Jennings, Gilles Waton, François Schosseler, Eduardo Mendes

\section{To cite this version:}

Laurence Jennings, Gilles Waton, François Schosseler, Eduardo Mendes. Towards a Rational Morphology Control of Frozen Copolymer Aggregates. Soft Matter, 2017, 13 (36), pp.6090-6099. 10.1039/C7SM01086A . hal-03508665

\section{HAL Id: hal-03508665 https://hal.science/hal-03508665}

Submitted on 3 Jan 2022

HAL is a multi-disciplinary open access archive for the deposit and dissemination of scientific research documents, whether they are published or not. The documents may come from teaching and research institutions in France or abroad, or from public or private research centers.
L'archive ouverte pluridisciplinaire HAL, est destinée au dépôt et à la diffusion de documents scientifiques de niveau recherche, publiés ou non, émanant des établissements d'enseignement et de recherche français ou étrangers, des laboratoires publics ou privés. 


\title{
Journal Name
}

\section{ARTICLE TYPE}

Cite this: DOI: $10.1039 / x x x x x x x x x x$

\section{Towards a Rational Morphology Control of Frozen Copolymer Aggregates}

\author{
Laurence Jennings, ${ }^{* a}$ Gilles Waton, ${ }^{a}$ François Schosseler ${ }^{* a}$ and Eduardo Mendes ${ }^{b}$
}

Received Date

Accepted Date

DOI: 10.1039/xxxxxxxxxx

www.rsc.org/journalname

\begin{abstract}
Kinetically frozen copolymer micelles are commonly prepared by confining amphiphilic block copolymers in the evaporating dispersed phase of oil-in-water emulsions. We revisit the mechanisms of this process by examining its successive steps separately: the formation of the solvent/water interface, the emulsification, the solvent evaporation and the formation of the aggregates. We bring into evidence that: i) Spontaneous water in solvent emulsification, i.e., the formation of a double emulsion, is a necessary step for the subsequent assembly of the copolymers into kinetically frozen aggregates with certain morphologies far from equilibrium. ii) Equilibration of the copolymer conformation at the solvent-water interfaces is a relatively slow process that can be outpaced, or even quenched before completion, by fast solvent evaporation rates. iii) Rather than dictated by the packing parameter at equilibrium, the morphology of the aggregates is determined by the effective copolymer conformation at the solvent-water interface when they form. iv) Ultra-long worm-like micelles do not form by a direct digitation of the dispersed oil phase into the water continuous phase but through the inversion of the double emulsion. From these findings, we design a simple setup that allows to control the morphology of the frozen aggregates obtained from a given copolymer composition by simply tuning the solvent evaporation rate.
\end{abstract}

\section{Introduction}

Surfactant micelles are generally defined by their dynamic nature involving a constant exchange of surfactant molecules between each other. The exchange kinetics are largely dominated by the strength of the incompatibility between the solvent and the insoluble surfactant molecule moiety. For surfactant molecules or amphiphilic copolymers with a large enough molecular weight, kinetics can become so slow that micelles cannot form by direct dissolution in a block selective solvent.

Eisenberg et al. demonstrated the concept of kinetically frozen polymer micelles and showed the wide range of kinetically stable morphologies obtained by progressively adding water to solutions of strongly hydrophobic block copolymers in an organic solvent of both blocks. ${ }^{1-3}$ For high enough water fraction, unimer exchange is prevented and micelles with frozen morphology and fixed aggregation number are obtained.

\footnotetext{
${ }^{a}$ Université de Strasbourg, CNRS UPR 22, Institut Charles Sadron, 23 rue du Loess, 67034 Strasbourg cedex 2, France. Fax: 33 (0)3 884140 99; Tel: 33 (0)3 884140 39; E-mail: laurence.jennings@ics-cnrs.unistra.fr, francois.schosseler@icscnrs.unistra.fr

${ }^{b}$ Advanced Soft Matter Group, Department of Chemical Engineering, Delft University of Technology, Van der Maasweg 9, 2629 HZ Delft, The Netherlands.

$\dagger$ Electronic Supplementary Information (ESI) available: Microscopy images of the spontaneous emulsification process, SEM imags of the aggregates, LSCM of adsorbed droplets, and videos of aggregates formation. See DOI: 10.1039/b000000x/
}

A decade later, Zhu et al. introduced the emulsion evaporation method where the common solvent of both blocks is immiscible with water and constitutes the dispersed phase of an oil in water emulsion. By further evaporating the organic solvent, the confined copolymers can be assembled into kinetically frozen micelles of different morphologies and efficiently loaded with hydrophobic compounds or nanoparticles. ${ }^{4,5}$

Currently however, there is no clear understanding of the parameters governing the aggregation of the unimers into one or another morphology. Zhu et al. suggested that the local increase in copolymer concentration, due to the evaporation of the dispersed phase, decreases the interfacial tension between the two solvents, which could reach null or even transiently negative values, thus generating the driving force for the formation of out of equilibrium morphologies as a means to increase the total surface of the system. ${ }^{6}$

This interpretation is based on a theoretical model by Granek et al. which describes the spontaneous emulsification of oil droplets in a water solution of surfactant molecules. ${ }^{7}$ There are two main differences between the model and the experimental conditions. In the model the continuous phase is the reservoir of surfactant molecules; conversely, in the experimental case the dispersed phase contains the copolymers. Moreover, the model describes low molecular weight surfactants and links the characteristic size of the formed micelles to the characteristic length of the grow- 
ing fluctuations of the interface. The calculation of the latter does not include any specific effects associated with the constraints of chain conformation for high molecular weight block copolymers under confinement. ${ }^{7}$ Indeed, the confinement of amphiphilic block copolymers at a solvent-solvent interface affects its local curvature. ${ }^{8}$

Parallel to the explanation based on interfacial tension, $\mathrm{Su}$ et al. have shown that using solvents with different boiling points has a clear effect on the morphology of the assembled objects. ${ }^{9}$ Their results, together with previous reports on the budding of oblate vesicles, ${ }^{8}$ suggest that increasing surface coverage results in the formation of micelles by budding, tubing or fingering of the solvent/water interface due to changes in its local curvature rather than changes in its interfacial tension.

One puzzling and common feature of both solvent replacement and emulsion evaporation methods is the often observed coexistence of different frozen aggregates morphologies. ${ }^{4,10}$ This diversity decreases considerably the usefulness of these methods since potential applications, e.g., in pharmaceutical formulations of nano-carriers, require often a strict control on size and morphology of the aggregates.

Clearly the formation of kinetically frozen micelles by evaporating emulsion droplets involves both thermodynamic equilibrium parameters and kinetic aspects in obtaining specific morphologies. ${ }^{11,12}$ However our understanding of the relative importance of thermodynamics and kinetics is still empirical and will remain so until an attempt to vary them independently. This is the purpose of our present study.

We used a system with the minimal number of components, consisting in poly(styrene-b-ethylene oxide) (PS-PEO) copolymers dissolved in a chloroform phase in contact with a pure water phase. After evaporation of the organic phase, the resulting aggregates are dispersed in water and are kinetically stable due to the glassy hydrophobic PS core. We used two PS-PEO copolymers with respective PEO weight fraction of $65 \%$ and $30 \%$. These two copolymers, consistently with the packing parameter concept developed for small surfactants, ${ }^{13}$ form mainly spherical and elongated micelles, respectively, through the emulsion evaporation method. ${ }^{4,14}$

We studied the problem step-by-step by directly observing each key stage: from the initial formation of chloroform/water interfaces to the evaporation of the chloroform emulsion droplets and the formation of copolymer assemblies in water. Each stage was investigated for the two copolymer compositions. We show that we can control the morphology of the aggregates independently of the copolymer's block length ratio by simply tuning the evaporation rate of the organic solvent.

\section{Materials and methods}

\subsection{Materials}

Poly(styrene-b-ethylene oxide) block copolymers $\mathrm{PS}_{16 \mathrm{k}}-\mathrm{PEO}_{7.5 \mathrm{k}}$ $(\mathrm{PDI}=1.09)$ and $\mathrm{PS}_{9.5 \mathrm{k}}-\mathrm{PEO}_{18 \mathrm{k}}(\mathrm{PDI}=1.04)$ were purchased from Polymer Source Inc, Canada. The subscripts indicate the molecular weight of the blocks in Dalton. Anhydrous chloroform $\geq 99 \%, 0.5-1 \%$ ethanol as stabiliser, and the fluores- cent dye 1,1'-Dioctadecyl-3,3,3',3'-tetramethylindocarbocyanine perchlorate (DiI) were purchased from Sigma Aldrich. Quartz cuvettes were purchased from Hellma and made of Suprasil ${ }^{\circledR}$ quartz, with spectral range 200-2500 nm. The imaging chambers, CoverWell ${ }^{\circledR}$ Perfusion Chamber height x diameter: $2.5 \mathrm{~mm}$ x $9 \mathrm{~mm}$ and a volume of $150 \mu \mathrm{L}$, were purchased from Sigma Aldrich. Silicon chips $(5 \times 5 \mathrm{~mm})$ for the SEM experiments were obtained from SIL'TRONIX ST.

\subsection{Planar interface microscopy experiments}

We performed these experiments in quartz cuvettes placed in a thermostated holder $\left(25^{\circ} \mathrm{C}\right)$ and used a horizontal backilluminated microscope setup to observe the evolution of the chloroform/water interface with a 5x objective. An Infinity2 camera (Lumenera) captured the images.

\section{In the absence of evaporation}

We prepared $100 \mathrm{mg} / \mathrm{mL}$ stock solutions of each copolymer in chloroform. The initial dissolution was facilitated using a vortex mixer. The solution was subsequently left overnight to fully dissolve the block copolymer. Using a glass syringe $100 \mu \mathrm{L}$ of stock solution were placed on the bottom of a quartz cuvette (path length $1 \mathrm{~mm}$, volume $350 \mu \mathrm{L}$ ) and $100 \mu \mathrm{L}$ of water were immediately but gently added to the chloroform/air interface. The cuvette was immediately sealed with a PTFE cap and put into the thermostated holder.

\section{With controlled evaporation rate}

For these experiments the copolymer concentration in the stock chloroform solutions was $10 \mathrm{mg} / \mathrm{mL}$. We filled $1 \mathrm{~mL}$ of milliQ water into a $3.5 \mathrm{~mL} 1 \mathrm{x} 1 \mathrm{~cm}$ quartz cuvette and added then 10 $\mu \mathrm{L}$ of copolymer stock solution at the water-air interface using a glass syringe. We sealed the cuvette with a PTFE cap fitted with a syringe needle connected to a peristaltic pump REGLO Digital MS-2/8 (ISMATEC). The tubing used with the peristaltic pump had an inner diameter of $1.52 \mathrm{~mm}$. The cuvette was placed in the thermostated holder.

\subsection{Observation of emulsion destabilisation}

For these experiments we prepared a stock solution of $10 \mathrm{mg} / \mathrm{mL}$ PS-PEO in chloroform containing also $0.02 \mathrm{wt} \%$ of the fluorescent dye DiI. From this solution, $10 \mu \mathrm{L}$ were placed in a vial containing $1 \mathrm{~mL}$ of MilliQ water. An emulsion was formed using a vortex for 30 seconds. The emulsion was then transferred, using a glass syringe, into a perfusion chamber stuck onto a glass coverslip. The chambers were not sealed allowing solvent evaporation. Instability of the droplets occurred within a few minutes from the preparation of the perfusion chamber and was observed in real time with a Nikon Eclipse TE2000E inverted fluorescence microscope.

\subsection{Scanning Electron Microscopy}

Plasma treated silicon chips were coated with $5 \mu \mathrm{L}$ of the aqueous solutions containing the aggregates. These were imaged using a SU8000 UHR Cold-Emission FE-SEM Scanning Electron Mi- 


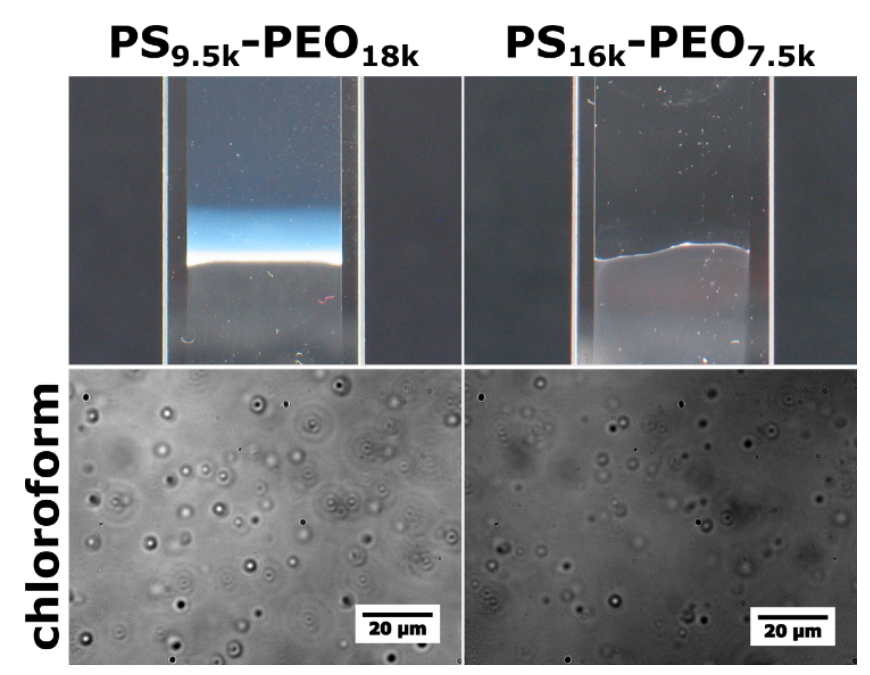

Fig. 1 Top row: close view of the interfacial region in the two cuvettes containing a PS-PEO in chloroform solution (bottom phase) in contact with water (upper phase). Bottom row: optical microcopy images of the PS-PEO/chloroform phase. All images were taken 24 hours after the formation of the interface.

croscope (Hitachi, Japan). The micellar samples were imaged at $1 \mathrm{kV}$ acceleration voltage without the addition of a conductive coating. The larger aggregates were sputtered with gold using a Bal-Tec SCF 050 Sputter Coater before imaging. A coating of approximately $10 \mathrm{~nm}$ was applied using a current of $40 \mathrm{~mA}$ and a sputtering time of 30 seconds at a working distance of $50 \mathrm{~mm}$.

\section{Results}

\subsection{Macroscopic planar interface without evaporation}

We studied first the emulsification process at a planar macroscopic interface without evaporation. According to the current model of spontaneous emulsification, $4,5,7$ this phenomenon should occur at high enough interface coverage by the copolymer. Therefore we chose a rather high initial copolymer concentration $(100 \mathrm{mg} / \mathrm{mL})$ to observe it in the absence of evaporation. We built carefully the planar interface in a sealed cuvette as described in section Materials and Methods. The upper row of Fig. 1 shows views of the biphasic systems obtained with the two copolymers 24 hours after the formation of the interface. The second row shows microscopy images of the bottom PS-PEO/chloroform phase.

With this system the emulsion evaporation method yields aqueous suspensions of copolymer aggregates with a glassy PS core and a PEO corona. Based on the current understanding of this method, ${ }^{4-7}$ we would therefore expect to observe spontaneous formation of a chloroform in water emulsion in Fig. 1.

Instead, both copolymers initially formed spontaneously water in chloroform emulsions, with water droplets still clearly visible after 24 hours (Fig. 1, 2nd row). This formation of water droplets in the chloroform phase occurs almost immediately after the two solvents are placed in contact (Fig. S1 and Movie $1^{\dagger}$ ).

The spontaneous formation of water in oil emulsions in similar systems is a known phenomenon and various mechanisms for it were reviewed in the literature. ${ }^{15,16}$ These include the above mentioned transiently null or negative interfacial tension, or the presence of impurities driving osmotic transport of water to oil containing salt impurities. ${ }^{17}$ In the present case, however, the occurrence of interfacial turbulence driven by, e.g., concentration gradients ${ }^{15}$ seems a more likely mechanism in view of the strong flow of water droplets through the water/chloroform interface (Movie $1^{\dagger}$ ).

Only after several hours, a bluish colloidal suspension appeared in the water phase of the sample containing $\mathrm{PS}_{9.5 \mathrm{k}}-\mathrm{PEO}_{18 \mathrm{k}}$ (Fig. 1, top left) and optical microscopy confirmed the presence of chloroform droplets in this phase. After evaporation of a small quantity of the upper transparent water region, scanning electron microscopy confirmed the formation of spherical micelles from the water phase for this sample (Fig. S2 ${ }^{\dagger}$ ). Thus upon formation of the macroscopic interface with $\mathrm{PS}_{9.5 \mathrm{k}}-\mathrm{PEO}_{18 \mathrm{k}}$ dissolved in chloroform, two phenomena occur at the interface on very different time scales: a violent mixing creating water droplets in the chloroform phase and a much slower emulsification of chloroform droplets in the water phase. Interfacial tension measurements on evaporating droplets showed that micelles form once the copolymer concentration reaches a certain value, independently of the copolymer's molecular weight or block length ratio. ${ }^{6}$ Thus our results suggest that this critical copolymer concentration at the macroscopic interface is reached only after a few hours in the absence of evaporation.

In the case of $\mathrm{PS}_{16 \mathrm{k}}-\mathrm{PEO}_{7.5 \mathrm{k}}$, no chloroform droplets formed in the upper water phase contrary to our expectations based on the current understanding of micelle formation by the emulsion evaporation method. ${ }^{4-7}$

These observations indicate a fundamental difference between the formation pathway of spherical or elongated micelles. The latter cannot form through interfacial destabilisation induced by null or transiently negative interfacial tension due to copolymer accumulation at the interface, as proposed in earlier reports. ${ }^{4-7}$

\subsection{Micelle formation by emulsion evaporation}

In light of these findings, we examine now the more complex conditions of evaporating emulsion droplets. Using a vortex, we prepared chloroform in water emulsions with droplets having diameters between 5 and $20 \mu \mathrm{m}$. A small amount of hydrophobic DiI dye added to the chloroform allowed the observation of droplet destabilisation by fluorescence microscopy. An overview of the results is shown in Fig. 2 and videos for each situation are available (Movies 2-5 ${ }^{\dagger}$ ). In the case of spherical micelles, fluorescence microscopy showed only shrinking droplets and the presence of micelles with spherical morphology was confirmed by SEM on the dried sample (Fig. 2, top left). For all the other cases, Fig. 2 shows snapshots extracted from movies shot during the formation of the aggregates. The floating emulsion droplets formed micelle morphologies in agreement with those reported in the literature. ${ }^{4,14}$ However, a fraction of the droplets, which were adsorbed on the glass slide of the imaging chamber, formed objects of lower curvature: elongated micelles for $\mathrm{PS}_{9.5 \mathrm{k}}-\mathrm{PEO}_{18 \mathrm{k}}$ and vesicles, implying bilayer formation, for $\mathrm{PS}_{16 \mathrm{k}}-\mathrm{PEO}_{7.5 \mathrm{k}}$.

Scanning electron microscopy of the aggregates formed from 
Floating
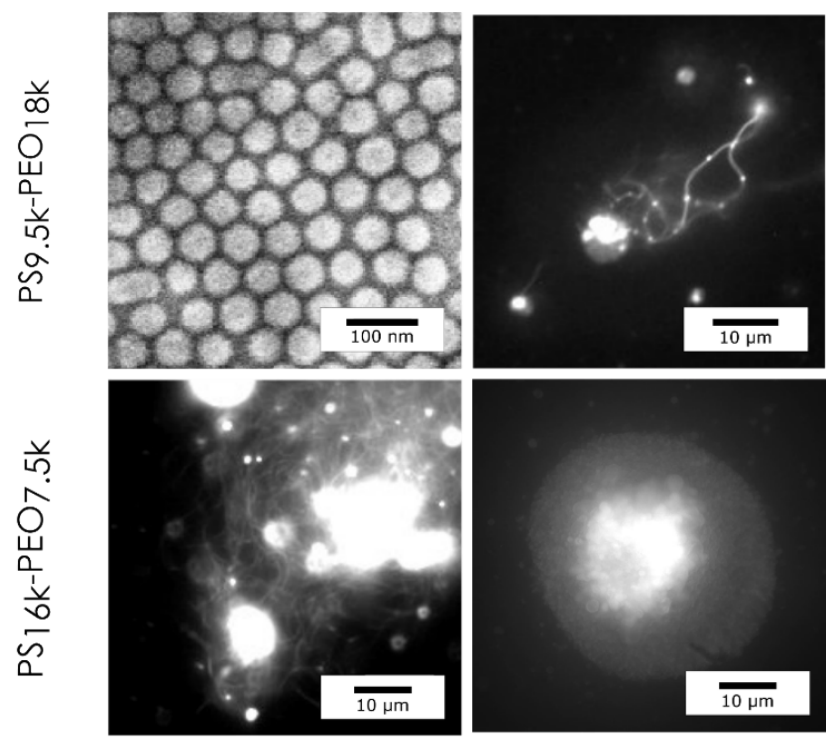

Fig. 2 Micelle morphologies formed during the evaporation of floating (left) or adsorbed (right) droplets of chloroform containing $\mathrm{PS}_{9.5 \mathrm{k}}-\mathrm{PEO}_{18 \mathrm{k}}$ (top) or $\mathrm{PS}_{16 \mathrm{k}}-\mathrm{PEO}_{7.5 \mathrm{k}}$ (bottom): spherical micelles (top left, SEM), elongated micelles (top right and bottom left), vesicles (bottom right).

adsorbed droplets containing $\mathrm{PS}_{16 \mathrm{k}}-\mathrm{PEO}_{7.5 \mathrm{k}}$, confirmed the presence of budding vesicles and strings of vesicles (Figs. S3 and $\left.S 4^{\dagger}\right)$. Zhu et al reported the formation of similar morphologies from chloroform droplets containing PS-PEO and SDS. ${ }^{18}$ However, from purely topological considerations, the formation of these structures is not fully compatible with the spontaneous emulsification theory. ${ }^{7}$ While a fingering instability of the polymer monolayer at the emulsion interfaces can yield spherical or elongated micelles, interconnected bilayers (multi-vesicles and strings of vesicles) cannot form without a series of folding deformations (Fig. 3). These are especially unlikely to occur if their driving forces are low interfacial energies or spontaneous curvatures.

On the other hand, the presence of smaller water droplets in the chloroform droplets, i.e., a double emulsion, would naturally yield these topologies (Fig. 3, bottom). The formation of such a double emulsion after the initial chloroform in water emulsification could happen either through violent interfacial mixing as described above for the planar interface or through osmotic transport. ${ }^{17}$

Investigating the fine structure of emulsion droplets was not possible in the case of copolymer $\mathrm{PS}_{9.5 \mathrm{k}}-\mathrm{PEO}_{18 \mathrm{k}}$. However for $\mathrm{PS}_{16 \mathrm{k}}-\mathrm{PEO}_{7.5 \mathrm{k}}$, we could remove abruptly the more volatile organic solvent by vacuum drying. This procedure allowed us to recover intermediate structures of the chloroform droplets frozen before their destabilisation. The frozen droplet structure looks like a "ball of yarn" of interconnected domains prior to the formation of elongated micelles (Fig. 4). Therefore, elongated structures form already within the chloroform droplets before these become visibly unstable. These frozen structures are reminiscent

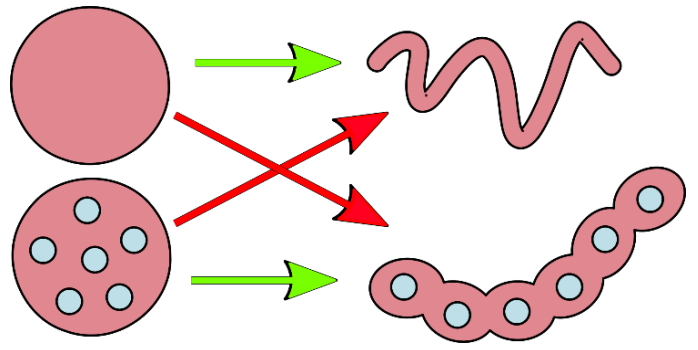

Fig. 3 Schematic formation of elongated micelles and strings of vesicles (right) from a single or multiple emulsion droplet (left). Stretching the interface of a single emulsion droplet (top) can yield a cylindrical structure without tearing or rupturing the interface. Similarly, stretching a multiple emulsion droplet (bottom) can yield a string of vesicles. Other transformations (red arrows) would require tearing the interface.

of a complex arrangement of entangled chloroform and water domains in the evaporating chloroform droplets.

The scheme in Fig. 5 summarises the effects of our experimental conditions on the aggregates morphology. The left column shows the case of the planar interface without any solvent evaporation. The two right columns present the results for floating or adsorbed droplets with evaporation. In each row, for a given copolymer composition, the aggregates morphology depends on the evaporation conditions and on the adsorption state of the droplets, i.e., physical parameters override copolymer composition in determining the aggregates morphology. To our knowledge, this is the first report of such effects. This finding could explain the polymorphism commonly observed in samples formed through the emulsion evaporation method. Fig. $\mathrm{S}^{\dagger}{ }^{\dagger}$ and Movies 6-7 $7^{\dagger}$ show typical 3D reconstructions of confocal slices imaged close to the bottom of the imaging chamber, before and after the micelle formation. The polymorphism observed in a given sample will likely depend on the effective surface/volume ratio in the preparation vessel.

\subsection{Controlling aggregate morphology through the evapora- tion rate}

To distinguish the effects of the adsorption state of the droplets from those of evaporation, we designed a new experiment: depositing a single droplet of chloroform at the air/water interface we controlled the solvent evaporation using a peristaltic pump (Fig. 6, top). With this setup we eliminate any adhesion and interaction of the solvent with the glass vial.

Chloroform has a higher density than water $(1.489 \mathrm{~g} / \mathrm{mL}$ at $25^{\circ} \mathrm{C}$ ), but, at $25^{\circ} \mathrm{C}$ and without the addition of any surfactants, the surface tension of chloroform with air is $26.7 \mathrm{mN} / \mathrm{m}$ which is smaller than that of water with air: $72 \mathrm{mN} / \mathrm{m} .{ }^{19,20}$ This makes it possible to deposit and balance chloroform droplets onto a water surface, provided that their mass is small enough to be balanced by the energy gained from the formation of the chloroform/air interface. ${ }^{21}$ All the above continues to be possible when using a chloroform solution containing PS-PEO copolymers.

We used three increasing flow rates from the pump. Since the nominal flow values for the pump are only applicable to liquids, we denote the flow rates used herein as $\mathrm{x} 1, \mathrm{x} 2$ and $\mathrm{x} 6$ (Fig. 7) 

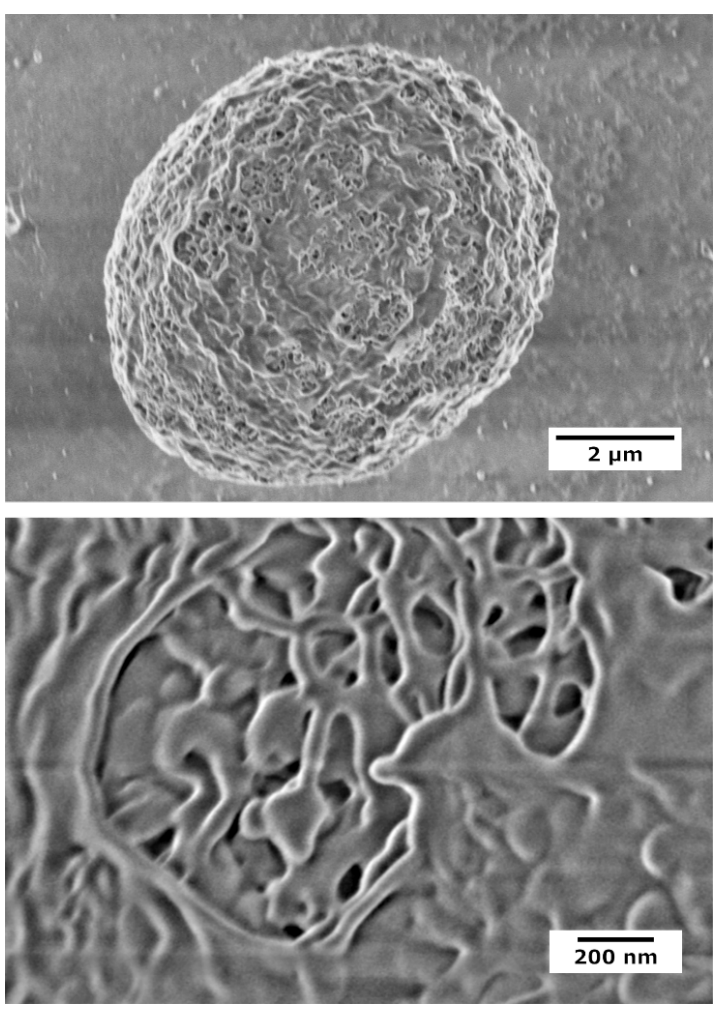

Fig. 4 SEM image of a dry emulsion droplet of chloroform containing $\mathrm{PS}_{16 \mathrm{k}}-\mathrm{PEO}_{7.5 \mathrm{k}}$ : an entangled structure suggests a complex arrangement of chloroform and water domains in the evaporating chloroform droplets.

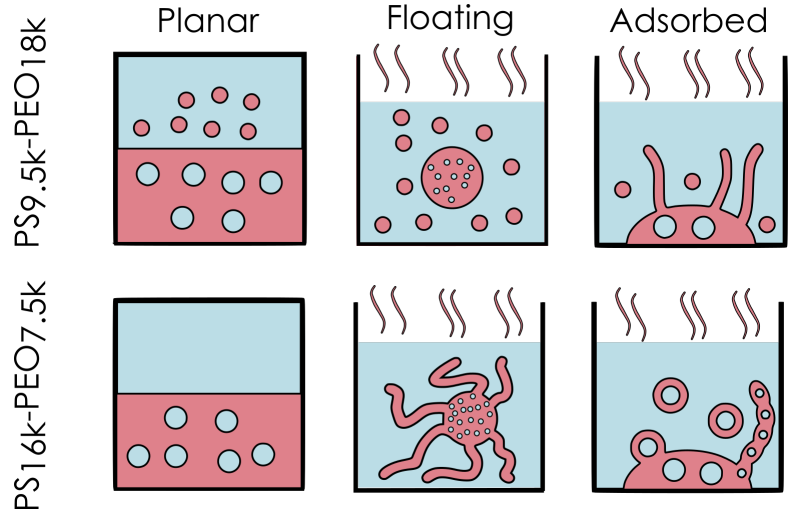

Fig. 5 Schematic overview of the morphologies obtained with planar interfaces, floating and adsorbed emulsion droplets.

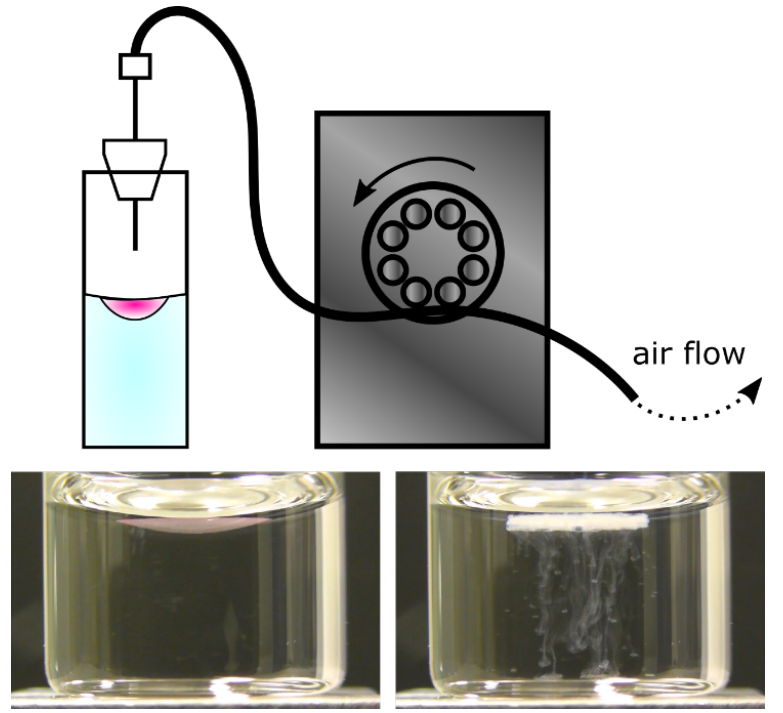

Fig. 6 (Top) Scheme of the experimental setup: a sealed cuvette, filled with water and a droplet of chloroform/copolymer solution deposited at the water air interface, is connected to a peristaltic pump extracting vapour. (Bottom) Photographs of a cuvette before (left) and during (right) the formation of the micelles. The fluorescent dye Dil was added to the $\mathrm{PS}_{16 \mathrm{k}}-\mathrm{PEO}_{7.5 \mathrm{k}} /$ chloroform solution to enhance its visibility.

indicating their relative increase. Indeed, under these conditions the $10 \mu \mathrm{L}$ chloroform droplet was evaporated in approximately 40,20 or 7 minutes, respectively.

As soon as the chloroform is deposited onto the water, a water in chloroform emulsion starts forming. During the evaporation of the organic solvent we observe a continuous change in the surface area of the chloroform droplet. Both the reduction of the solvent's mass and the decrease of its surface tension with water provide a driving force for a spreading of the chloroform droplet.

When using the $\mathrm{x} 1$ flow rate, the chloroform droplet shrinks and spreads on the water surface for the first 40 minutes. After this stage spherical micelles are present in the water phase when using the $\mathrm{PS}_{9.5 \mathrm{k}}-\mathrm{PEO}_{18 \mathrm{k}}$. With the more hydrophobic $\mathrm{PS}_{16 \mathrm{k}}-\mathrm{PEO}_{7.5 \mathrm{k}}$ that forms elongated micelles, we observe an abrupt transition from a thin chloroform layer at the water surface into a series of filaments growing towards the bottom of the vial. Fig. 6 shows two snapshots taken before (bottom left) and after (bottom right) the formation of the elongated micelles.

After the total evaporation of the organic solvent we performed SEM to image the objects formed in the water phase (Fig. 7). At the lowest solvent evaporation rate, each copolymer formed the same morphologies as reported in the literature ${ }^{4,14}$ and as expected from their packing parameter: ${ }^{13}$ spherical for the more hydrophilic copolymer and elongated for the more hydrophobic one (top row). This suggests that the lowest evaporation rate provides enough time for the copolymers to rearrange into a conformation consistent with their packing parameter. Increasing the evaporation rate we observe a transition from spherical micelles to elongated ones for the $\mathrm{PS}_{9.5 \mathrm{k}}-\mathrm{PEO}_{18 \mathrm{k}}$ and a transition from elongated micelles to vesicles for the $\mathrm{PS}_{16 \mathrm{k}}-\mathrm{PEO}_{7.5 \mathrm{k}}$ (middle row). Finally, at the highest evaporation rates we obtain vesicles for both the copolymers used (bottom row). The characteristic diameters of 

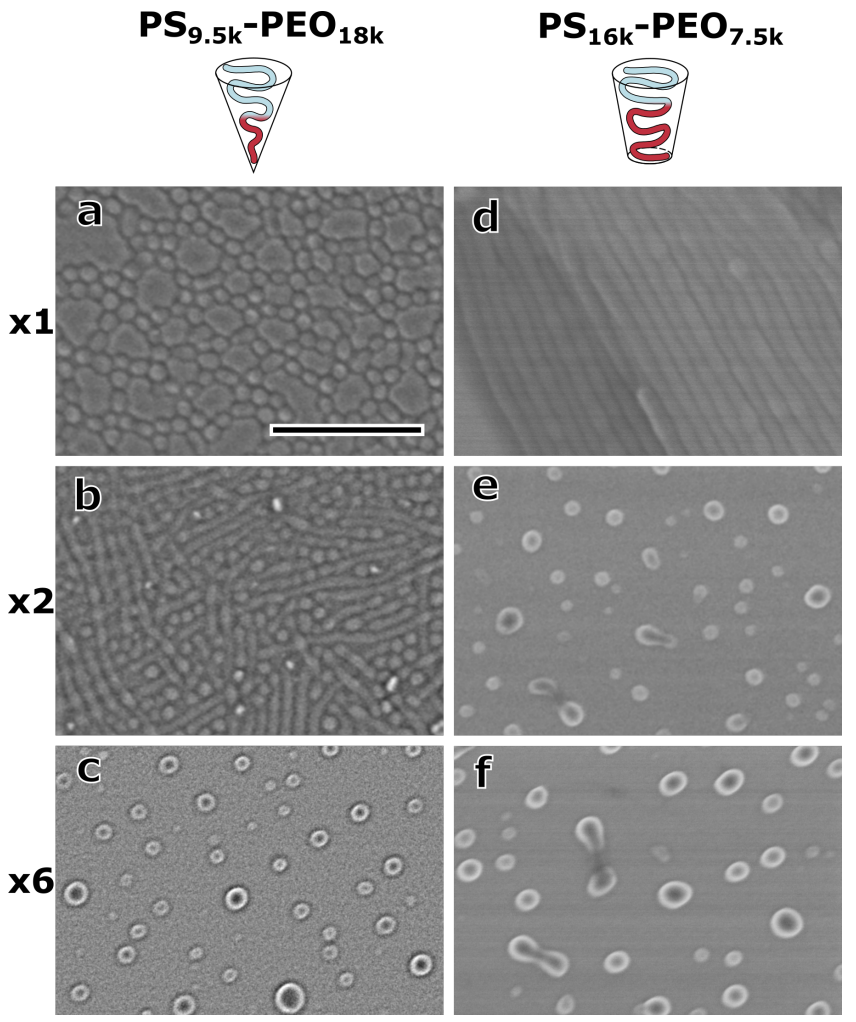

Fig. 7 Different morphologies obtained by varying the evaporation rate and visualised using Scanning Electron Microscopy. On the left $\mathrm{PS}_{9.5 \mathrm{k}}-\mathrm{PEO}_{18 \mathrm{k}}$ and on the right $\mathrm{PS}_{16 \mathrm{k}}-\mathrm{PEO}_{7.5 \mathrm{k}}$. Ultimately both copolymers lead to similar vesicular morphologies. a) spherical micelles; b) elongated and spherical micelles; d) elongated micelles; c), e) and f) vesicles. Scale bar $500 \mathrm{~nm}$.

the aggregates were estimated from SEM images and are listed in Table 1.

Table 1 Morphologies and average diameters $(\mathrm{nm})$ obtained as a function of the pump flow rate. The letters indicate the morphology of the aggregates: $S$ for spherical, $E$ for elongated and $V$ for vesicles. When two morphologies are present the more frequent is listed as first.

\begin{tabular}{llll}
\hline Polymer & x1 & x2 & x6 \\
PS $_{9.5 \mathrm{k}}-\mathrm{PEO}_{18 \mathrm{k}}$ & S: $45 \pm 5$ & E/S: $33 \pm 3 / 42 \pm 4$ & V: $58 \pm 18$ \\
PS $_{16 \mathrm{k}}-\mathrm{PEO}_{7.5 \mathrm{k}}$ & E: $55 \pm 5$ & V: $56 \pm 16$ & V: $72 \pm 18$ \\
\hline
\end{tabular}

The trend of a decreasing object curvature with increasing evaporation rate is consistent with the results obtained by $\mathrm{Su}$ et al. ${ }^{9}$ In the latter study however, the evaporation rate was varied by choosing solvents with different boiling temperature. Here our conclusion is stronger since the solvent quality remains the same. The observed effects hint to the importance of the relative rates of copolymer diffusion to the interface, conformational rearrangement at the interface, and solvent evaporation.

At the higher evaporation rates, yielding vesicles in the water phase, part of the initial chloroform droplet solidified into one single dome-like aggregate. This is easily understood: chloroform acts as a plasticiser for the PS blocks allowing the copolymers to rearrange their conformation at the solvent/water interface and to form vesicles. However, for low enough chloroform content, mobility is finally prevented and a frozen single aggre-
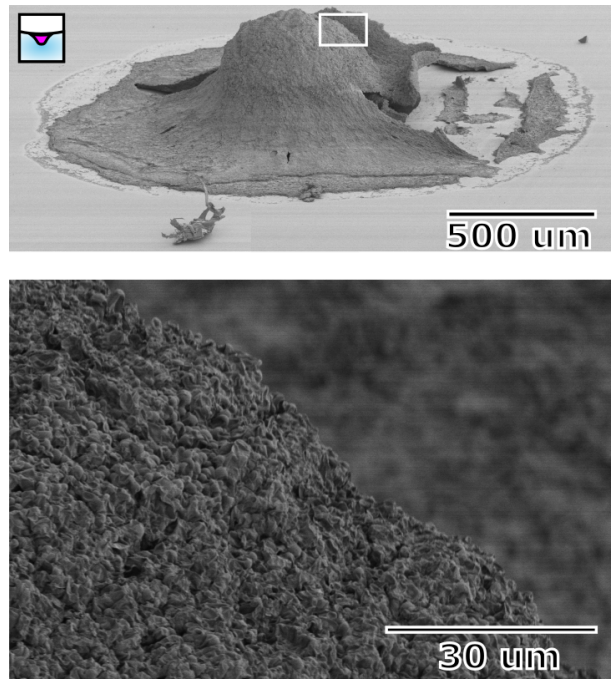

Fig. 8 SEM image of a budding vesicle aggregate formed at high evaporation rate. Top: Overview of the aggregate formed as a pending droplet at the air/water interface (insert); Bottom: Detail of the surface populated with spherical aggregates.

gate remains in the later stage.

Further inspection of these aggregates by SEM, Fig. 8, showed that they have a rough surface reminiscent of the budding vesicles shown by Zhu et al. ${ }^{22}$ Comparing with Fig. 4, there is a clear correlation between the structure of the dried droplets and the morphology of the formed aggregates in each case. These structures are only possible because of the initial presence of a water in chloroform emulsion which remains confined in the fast evaporating chloroform droplet.

\section{Discussion}

4.1 Copolymer conformation at water/chloroform interface We can explain the surprising formation of water in chloroform emulsions, irrespectively of the copolymer composition, by considering the peculiar solubility of PEO in water. PEO is generally assumed to be a hydrophilic polymer. This is indeed true only for low molecular weights $(<2000 \mathrm{~g} / \mathrm{mol})$ when the $\mathrm{OH}$ termination provides a strong contribution to water solubility. ${ }^{23}$ For higher molecular weights however, methanol, DMSO, chloroform and THF are increasingly better solvents for PEO than water. ${ }^{24}$

The better solubility of PEO in organic solvents is due to the presence of the $\mathrm{CH}_{2}$ groups, which contribute significantly to the polymer's hydrophobicity. However the backbone oxygens can also bind through hydrogen bonds to water molecules with minimal disturbance of the tetrahedral arrangement of bulk water. ${ }^{25}$ This combination causes PEO to be a weakly amphiphilic polymer on its own. ${ }^{26}$ An organic solvent capable of donating H-bond, like chloroform, can accommodate both the $\mathrm{CH}_{2}$ and $\mathrm{O}$ moieties in the PEO backbone. Therefore, pure chloroform is a better solvent for PEO than pure water. ${ }^{23}$ Nevertheless, the intrinsic strength of the $\mathrm{H}$-bond with chloroform is much weaker than the one with water. ${ }^{27}$ Therefore the better location for the PEO block should be along any interface between chloroform and water where both the $\mathrm{CH}_{2}$ groups and the $\mathrm{O}$ atoms have their preferred environ- 

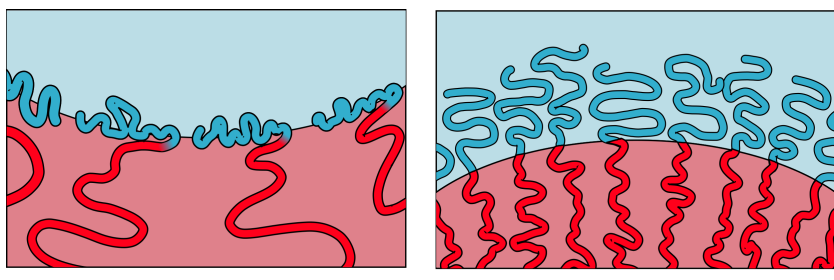

Fig. 9 Schematic representation of the copolymer conformation at the water/chloroform interface under low (left) and high (right) surface concentration.

ment.

As far as we know, no report investigated the conformation of PS-PEO copolymers at the chloroform/water interface. Earlier studies of these copolymers at a liquid-liquid interface focused on the toluene/water, ${ }^{28,29}$ the heptane/water, ${ }^{30}$ or the cyclohexane/water ${ }^{31}$ interfaces. None of these interfaces can mimic our experimental conditions where the organic phase is a good solvent for both blocks.

The best approximations we could find are studies of poly(styrene oxide)-PEO copolymers at water/chloroform interface, ${ }^{32}$ and of PEO homopolymers at water/dichloromethane interface. ${ }^{33,34}$ In both cases, results hint to a spreading of the PEO chains at the interface. Upon compression of the interfacial area, the interfacial films made of PEO show fast reorganisation and low energetic dissipation, consistent with the expulsion of a limited number of chain segments in the water phase, likely in the vicinity of the hydroxy end-groups. ${ }^{33,34}$ For the copolymers the surface activity is decreasing (lower surface coverage) with increasing hydrophobicity: rather large bulk concentrations are necessary to obtain a dense surface coverage and lower significantly the interfacial tension. ${ }^{32}$ Although the molecular weight and composition of our samples do not match those of these studies, the general trends should remain the same.

Therefore the conformation of the copolymers at the interface could be likened to a large floating PEO raft with a dangling PS tail extended in the chloroform phase, Fig. 9 left. In this conformation, the PEO favours a flat interface while the PS block promotes a convex curvature towards the chloroform phase. It becomes then clear that at low surface coverage the local curvature induced by the copolymers sitting at the interface promotes the formation of a water in oil emulsion as opposed to the higher surface coverage brush that favours the formation of a chloroform in water emulsion, Fig. 9 right.

The behaviour of copolymers at interfaces is often rationalised by introducing the spreading parameter concept. This is the difference between the work of adhesion and the work of cohesion of a substance at an interface and provides an idea of its wetting properties on a given surface. ${ }^{35}$ This parameter depends on the block copolymer block length ratio and has been linked to the morphology of micelles formed at the water-air interface. ${ }^{36}$ Different surface conformations of the copolymers will affect the spreading parameter by changing the contribution of the blocks to the adhesion and the cohesion at the interface. Therefore, when the interfacial coverage increases, e.g., by evaporation, and drives a conformational transition at the interface, the spreading parameter depends on the instantaneous adsorption state of the copolymers at the interface.

Clearly, the spreading parameter becomes a dynamic concept in the case of the non-equilibrium phenomena considered here. Its instantaneous value depends on the respective rates of surface coverage variation and interfacial conformation equilibration.

\subsection{Formation of the aggregates}

In this section, we discuss how the concept of a dynamical spreading parameter can explain the results in the different experimental conditions probed in this work.

\section{Planar interface without evaporation.}

Several mechanisms contribute to the dynamics of interfacial concentration in the absence of evaporation (Fig. 10), the most obvious one being the ordinary diffusion of bulk copolymers to the interface.

Water making contact with the chloroform surface results in a chemical potential shock. Since the initial copolymer surface concentration is at an equilibrium corresponding to the surface tension of chloroform $(\approx 26 \mathrm{mN} / \mathrm{m})$ and the interfacial tension for chloroform/water is much higher $(\approx 32 \mathrm{mN} / \mathrm{m})$, turbulent convection appears as an initial equilibration mechanism of the interface that is more efficient than diffusion. Clearly the experimental evidence (Movie $1^{\dagger}$ ) shows that this convection brings water droplets into the chloroform phase, which we attribute to a spontaneous interfacial curvature convex towards the chloroform phase as discussed above.

The creaming of water droplets brings copolymer chains back to the macroscopic flat interface. On the other hand, spontaneous water in chloroform emulsification depletes the copolymer concentration at this interface and slows down the increase of interface coverage. Once the instantaneous spreading parameter has reached a critical value, spontaneous chloroform in water emulsification can start if the copolymer composition is consistent with a convex curvature towards the water phase. This explains the delay between the formation of the interface and the formation of chloroform droplets in the water phase. For the more hydrophobic copolymer however, this never happens: the local interfacial curvature never reaches values allowing the digitation of chloroform into the water phase.

\section{Emulsion evaporation.}

The scheme in Fig. 11 describes the evaporation driven evolution of a solvent droplet (pink) in water (blue). For clarity, the droplet states are drawn the same size and the amount of copolymer (dark red) increases to mimic the concentration increase with evaporation (from top left to bottom right). We depict a situation where the copolymer composition is consistent with the formation of elongated micelles and the evaporation rate is slow compared to the conformation equilibration rate.

Initially, a double w/o/w emulsion is formed (Fig. 11, 1st row) following the mechanisms described in Fig. 10. For the more hydrophobic copolymers considered here, the curvature at low surface density is higher and favours the emulsification of smaller water droplets in the chloroform droplets, hence their cream- 


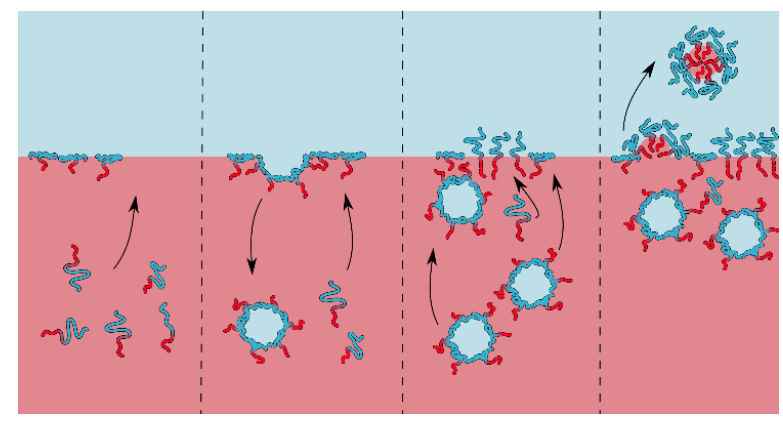

Fig. 10 Competing equilibration mechanisms at the planar interface in the absence of evaporation. From left to right: Copolymer diffusion from bulk to the interface; Water droplet formation in the chloroform phase, by either convection or spontaneous emulsification; Creaming of water droplets to the interface; Formation of chloroform in water droplets at high surface coverage.

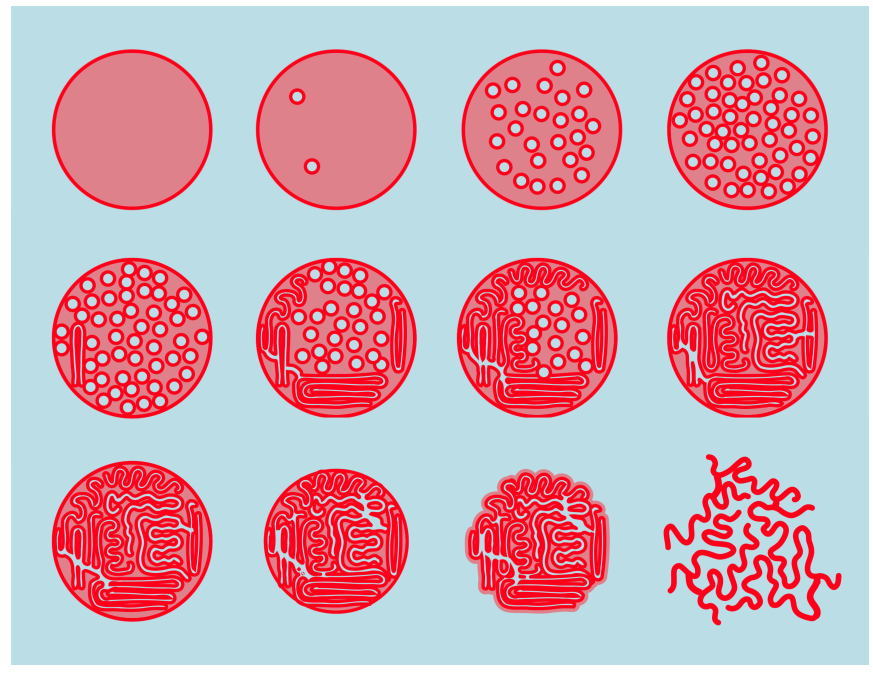

Fig. 11 Formation of elongated micelles from an evaporating solvent droplet (light red) in water (blue). Copolymers at interfaces pictured in dark red. From top left to bottom right the bulk copolymer concentration is increasing by evaporation. For clarity, the droplet states are drawn with the same size. See text for details. ing should be slower. As water droplets form and chloroform evaporates, both the water fraction and the copolymer concentration increase in the chloroform droplets until the phase inversion composition is reached. ${ }^{37}$ At that time, in slow evaporation conditions, the copolymers have adopted a dense brush conformation at the interfaces and the obtained morphology corresponds to their composition, i.e., to elongated micelles in the case depicted here. These newly formed elongated micelles are swollen with chloroform for the initial stage. However, the removal of this solvent will cause the PS blocks to rearrange within the micelle core. This has the tendency to stretch out and to stiffen the glassy PS-PEO micelles, which gives the impression that the micelles are being ejected from the evaporating chloroform droplet (Movies $\left.2,5^{\dagger}\right)$.

If the solvent is removed abruptly, the full inversion is prevented and the final morphology of the droplet is a ball of yarn of entangled elongated structures as shown in Fig. 4.

In the case of more hydrophilic copolymers, the solvent evaporation results in the same spherical morphology as obtained in the absence of evaporation as long as it is slow enough. This is simply because the direct formation of spherical micelles in the water phase is still possible. However the final diameter of the micelles can depend on the evaporation rate in this regime of slow evaporation. ${ }^{38}$

\section{Planar interface with controlled evaporation rate.}

Considering a suspended chloroform droplet deposited at the air/water interface, there is first the formation of a water emulsion inside, as discussed above for the other experimental configurations.

Subsequent solvent evaporation increases the copolymer concentration at every water-chloroform interface inside the chloroform droplet. We hypothesise that the morphology of the aggregates is determined by the adsorption state of the copolymers at the onset of surface instability, once sufficient organic solvent has evaporated.

When the solvent evaporation is slow enough, the adsorption state of the copolymers assumes a dense, close to equilibrium, brush configuration. Therefore the formed micelles represent more closely the usual packing parameter of the copolymers estimated from their composition. At higher evaporation rates, this is no longer the case, the copolymers have a different spreading parameter and aggregates form with morphologies far away from the ones expected from their composition.

Thus, at intermediate evaporation rates, the hydrophilic $\mathrm{PS}_{9.5 \mathrm{k}}-\mathrm{PEO}_{18 \mathrm{k}}$ has not enough time to equilibrate a dense brush conformation associated with a high spontaneous interfacial curvature. Therefore it forms elongated micelles similar to the equilibrium ones obtained with the hydrophobic $\mathrm{PS}_{16 \mathrm{k}}-\mathrm{PEO}_{7.5 \mathrm{k}}$ at low evaporation rates. Finally, when the evaporation rate is sufficiently fast, surface instability is met even before the spreading parameter corresponding to elongated micelles is reached. This corresponds to a situation in which the PEO blocks are largely parallel to the chloroform/water interfaces and thin bilayer vesicles are formed by the outwards pressure of the water droplets being confined in a copolymer rich evaporating solvent. 

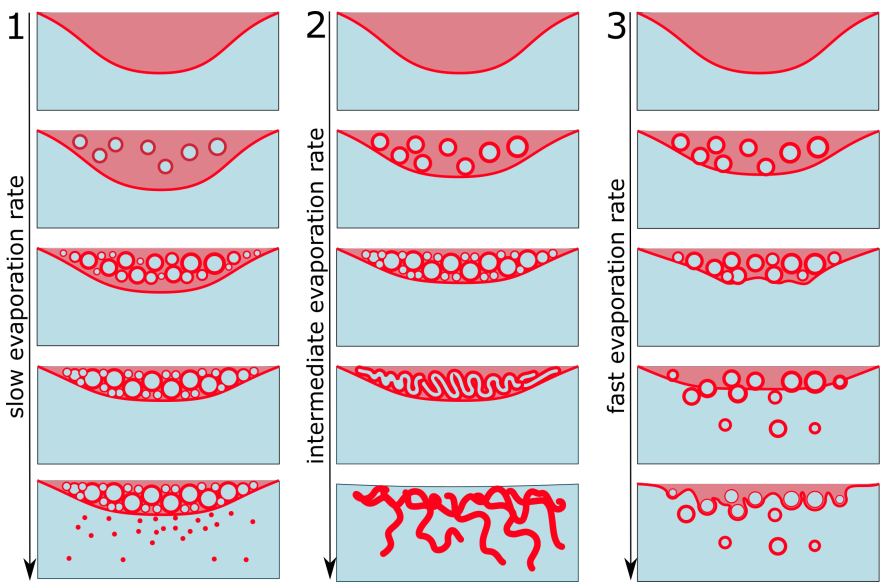

Fig. 12 Overview of the different formation mechanisms of the $\mathrm{PS}_{9.5 \mathrm{k}}-\mathrm{PEO}_{18 \mathrm{k}}$ micelles with increasing evaporation rate from left to right. Each column represents the evolution of copolymer in a chloroform droplet suspended on top of a water volume. The bottom row shows the final aggregates morphology: spherical (1) or elongated (2) micelles, vesicles (3), obtained with the increasing evaporation rates. See text for details.

Fig. 12 summarises the formation mechanisms for the hydrophilic $\mathrm{PS}_{9.5 \mathrm{k}}-\mathrm{PEO}_{18 \mathrm{k}}$ covering all the morphologies observed. It is worth emphasising that, except for slow enough evaporation rates, the aggregates form through a phase inversion induced by solvent evaporation. For the more hydrophobic $\mathrm{PS}_{16 \mathrm{k}}-\mathrm{PEO}_{7.5 \mathrm{k}}$, aggregates cannot form without the prior w/o emulsion formation and its phase inversion.

\subsection{Further comments}

Returning to the case of emulsion evaporation, in light of the proposed mechanisms, we can also explain the different aggregates morphologies obtained with floating and adsorbed droplets. The latter, having a higher surface to volume ratio than the floating ones (Fig. S5 ${ }^{\dagger}$ ), evaporate faster and do not produce morphologies consistent with the copolymer composition.

Micelles and vesicles obtained in our experiments display very similar characteristic sizes (Table 1). This is indeed possible only because the copolymer conformations in both types of objects are very different, strongly non-equilibrium ones in the case of vesicles. This makes it possible to obtain thin bilayers that seem at first sight inconsistent with the macromolecular dimensions deduced from the micellar sizes. However the size similarity between the micelles and the vesicles could still be simply coincidental.

Finally, we would like to point out that our last experimental geometry, the chloroform droplet deposited on water, creates the conditions for viscous fingering phenomena, which are observed when a less viscous liquid is pushed against a more viscous one. ${ }^{39}$ This phenomenon can also occur when the pressure is driven by gravity, e.g., when a less viscous higher density liquid layer is spread on a more viscous lower density layer. ${ }^{40}$ This is precisely the case when we place a chloroform droplet on top of water. In such a geometry, we can expect a wide variety of interesting phenomena, depending on the interfacial tension be- tween the two liquids. ${ }^{41}$ Thus viscous fingering could help the formation of elongated micelles in some conditions but does not invalidate the mechanisms proposed above. The investigation of the links between our results and viscous fingering would be very interesting but lies beyond the scope of our paper.

\section{Conclusions}

We studied the assembly of amphiphilic copolymers into kinetically frozen aggregates through the emulsion evaporation method. In addition to the common emulsion conditions, we made also experiments with a planar macroscopic interface, either without solvent evaporation or with controlled solvent evaporation rate.

From the observations of the interfacial behaviour by optical microscopy, we obtained three original insights about the mechanism of aggregate formation by the solvent evaporation method: i) spontaneous water in chloroform emulsification is a critical step for the subsequent formation of aggregates through emulsion inversion induced by solvent evaporation, ii) equilibration of the copolymer conformation at the interface is a relatively slow process that can be outpaced or even quenched before completion by fast evaporation rates, iii) morphology of the aggregates is determined by the effective copolymer conformation when emulsion inversion occurs.

We showed that, by controlling the solvent evaporation rate, we can form aggregates with morphologies different from the ones expected on the basis of copolymer composition.

Until now, the formation of pure block copolymer elongated micelles was restricted only to a very specific block length ratio of the copolymers. Our technique opens up new possibilities in the formulation of elongated micelles of very specific core/corona block length ratio. It provides the first steps towards the rational development of new approaches to control the morphology of micelles independently of their formulation.

Potential applications of these results can be foreseen in the field of nanomedecine where decoupling the respective sizes of hydrophobic core and hydrophilic corona can yield useful freedom in designing nano-carriers optimised for systemic circulation and cell penetration.

Furthermore, the previously unsuspected spontaneous emulsification step unveiled by our experiments opens new interesting perspectives for drug loading strategies.

\section{Acknowledgments}

We thank Dr. Marc Schmutz, from the electronic microscopy facility in Institut Charles Sadron, for his help with the SEM imaging. We thank also the reviewers whose comments helped to improve the original manuscript. The research leading to these results has received funding from the People Programme (Marie Curie Actions) of the European Union's Seventh Framework Programme (FP7/2007-2013) under REA grant agreement no. PITNGA-2012-317019 "TRACE and TREAT".

\section{References}

1 Z. Gao, S. K. Varshney, S. Wong and A. Eisenberg, Macromolecules, 1994, 27, 7923-7927. 
2 K. Yu and A. Eisenberg, Macromolecules, 1996, 29, 6359-6361.

3 L. Zhang and A. Eisenberg, J. Am. Chem. Soc., 1996, 118, 3168-3181.

4 J. Zhu and R. C. Hayward, J. Am. Chem. Soc., 2008, 130, 7496-7502.

5 W. Li, S. Liu, R. Deng and J. Zhu, Angew. Chem. Int. Ed., 2011, 50, 5865-5868.

6 J. Zhu and R. C. Hayward, J. Colloid Interf. Sci., 2012, 365, 275 - 279.

7 R. Granek, C. B. Robin and M. Cates, J. Physique II, 1993, 3, 829-849.

8 I. Tsafrir, Y. Caspi, M.-A. Guedeau-Boudeville, T. Arzi and J. Stavans, Phys. Rev. Lett., 2003, 91, 138102.

9 M. Su and Z. Su, Macromolecules, 2014, 47, 1428-1432.

10 L. Zhang and A. Eisenberg, Science, 1995, 268, 1728-1731.

11 R. C. Hayward and D. J. Pochan, Macromolecules, 2010, 43, 3577-3584.

12 M. Dionzou, A. Morere, C. Roux, B. Lonetti, J.-D. Marty, C. Mingotaud, P. Joseph, D. Goudouneche, B. Payre, M. Leonetti and A.-F. Mingotaud, Soft Matter, 2016, 12, 2166-2176.

13 J. N. Israelachvili, D. J. Mitchell and B. W. Ninham, J. Chem. Soc., Faraday Trans. 2, 1976, 72, 1525-1568.

14 P. J. Glazer, L. Bergen, L. Jennings, A. J. Houtepen, E. Mendes and P. E. Boukany, Small, 2014, 10, 1729-1734.

15 J. C. López-Montilla, P. E. Herrera-Morales, S. Pandey and D. O. Shah, J. Disper. Sci. Technol., 2002, 23, 219-268.

16 J. Bahtz, D. Z. Gunes, A. Syrbe, N. Mosca, P. Fischer and E. J. Windhab, Langmuir, 2016, 32, 5787-5795.

17 J. Bae, T. P. Russell and R. C. Hayward, Angew. Chem. Int. Ed., 2014, 53, 82408245.

18 J. Zhu, N. Ferrer and R. C. Hayward, Soft Matter, 2009, 5, 2471-2478.

19 N. Pallas and Y. Harrison, Colloids Surf., 1990, 43, 169 - 194.

20 A. W. Adamson and A. P. Gast, Physical Chemistry of Surfaces, Wiley Interscience, New York, 6th edn, 1997.

21 C. M. Phan, B. Allen, L. B. Peters, T. N. Le and M. O. Tade, Langmuir, 2012, 28, 4609-4613.

22 J. Zhu and R. Hayward, Angew. Chem. Int. Ed., 2008, 47, 2113-2116.

23 M. Spitzer, E. Sabadini and W. Loh, J. Braz. Chem. Soc., 2002, 13, 7 - 9.
24 C. Ö. Dinç, G. Kibarer and A. Güner, J. Appl. Polym. Sci., 2010, 117, 1100-1119.

25 R. Kjellander and E. Florin, J. Chem. Soc., Faraday Trans. 1, 1981, 77, 2053 2077.

26 J. Israelachvili, Proc. Nat. Acad. Sci. USA, 1997, 94, 8378-8379.

27 J. March, Advanced organic chemistry: reactions, mechanisms, and structure, McGraw-Hill, 1977.

28 R. M. Prokop, M. L. Hair and A. W. Neumann, Macromolecules, 1996, 29, 5902 5906.

29 G. Rother and G. H. Findenegg, Colloid Polym. Sci., 1998, 276, 496-502.

30 B. B. Sauer, H. Yu, C. F. Tien and D. F. Hager, Macromolecules, 1987, 20, 393 400.

31 D. E. Gragson, J. M. Jensen and S. M. Baker, Langmuir, 1999, 15, 6127-6131.

32 J. Juárez, S. Goy-López, A. Cambón, M. A. Valdez, P. Taboada and V. Mosquera, J. Phys. Chem. C, 2010, 114, 15703-15712.

33 A. Malzert, F. Boury, P. Saulnier, J. P. Benoît and J. E. Proust, Langmuir, 2002, 18, 10248-10254.

34 A. Malzert, F. Boury, P. Saulnier, T. Ivanova, I. Panaïotov, J. Benoît and J. Proust, J. Colloid Interf. Sci., 2003, 259, 398 - 407.

35 E. Lugscheider and K. Bobzin, Surf. Coatings Technol., 2003, 165, 51 - 57.

36 G. Li Destri, A. A. Malfatti Gasperini and O. Konovalov, Langmuir, 2015, 31, 8856-8864.

37 J.-L. Salager, A. Forgiarini, L. Márquez, A. Peña, A. Pizzino, M. P. Rodriguez and M. Rondón-González, Adv. Colloid Interf. Sci., 2004, 108-109, 259 - 272.

38 L. Jennings, O. Ivashchenko, I. J. C. Marsman, A. C. Laan, A. G. Denkova, G. Waton, F. J. Beekman, F. Schosseler and E. Mendes, Biomater. Sci., 2016, 4, 1202 1211.

39 D. Bensimon, L. P. Kadanoff, S. Liang, B. I. Shraiman and C. Tang, Rev. Mod. Phys., 1986, 58, 977-999.

40 J. Fernandez, P. Kurowski, P. Petitjean and E. Meiburg, J. Fluid Mech., 2002, 451, 239-260.

41 D. Bonn, H. Kellay, M. Ben Amar and J. Meunier, Phys. Rev. Lett., 1995, 75, 2132-2135. 


\section{Supporting Information}

\section{Towards a Rational Morphology Control of Frozen Copolymer Aggregates}

Laurence Jennings, Gilles Waton, François Schosseler and Eduardo Mendes

Table of Contents:

1. Microscopy images of the spontaneous emulsification process

2. Scanning Electron Microscopy images of the aggregates

3. Laser scanning confocal microscopy of adsorbed droplets

4. List of videos

Videos can be downloaded form the publisher's website. 


\section{Microscopy images of the spontaneous emulsification process}

A video of the spontaneous emulsification in presence of $\mathrm{PS}_{16 \mathrm{k}}-\mathrm{PEO}_{7.5 \mathrm{k}}$ can be downloaded from the publisher's website. Here is shown a montage containing four frames of the video taken 5 seconds apart from each other.

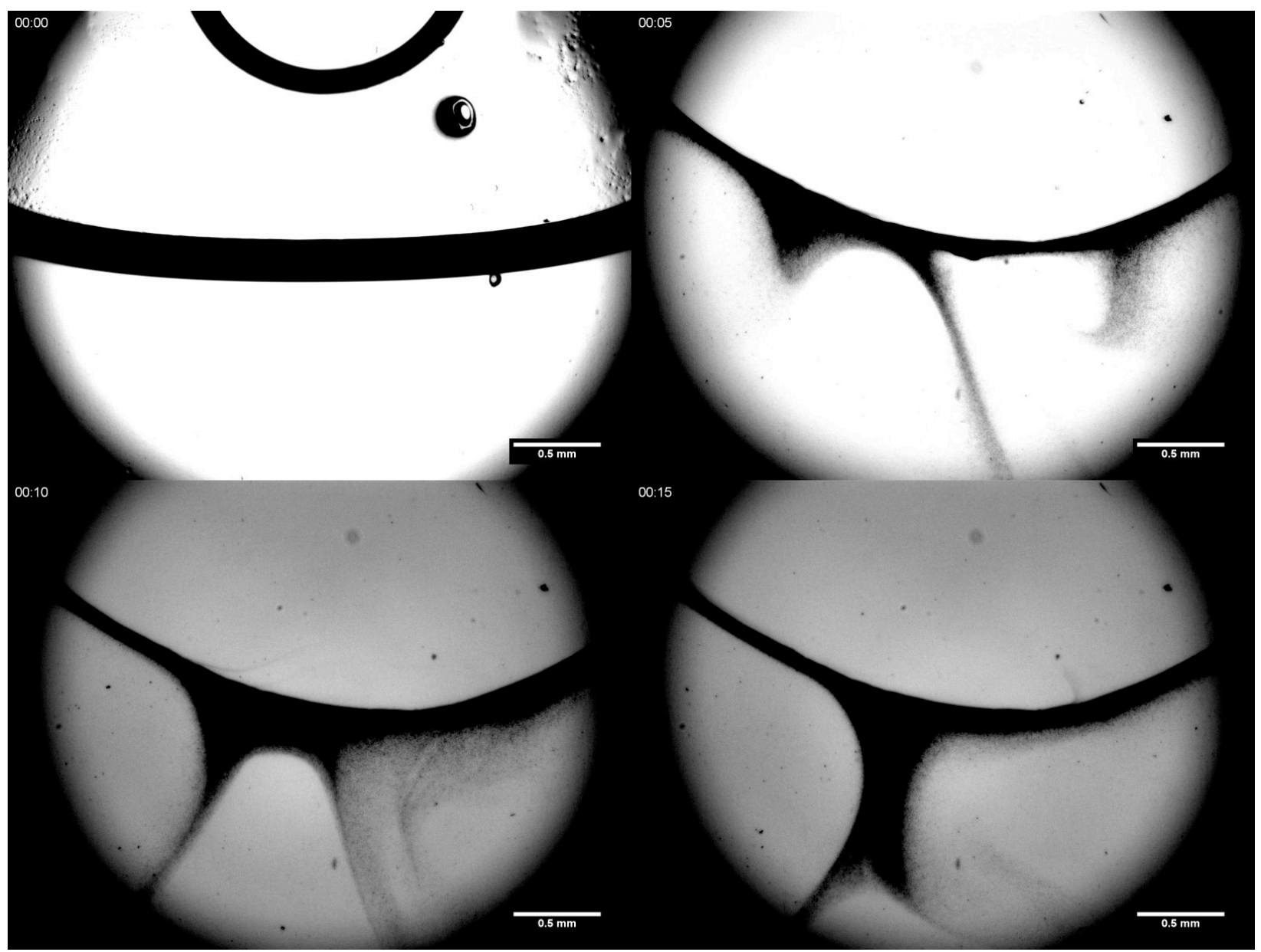

Figure S1. Montage from the video of spontaneous emulsification. From top left to bottom right each frame is taken 5 seconds after the previous one. In the initial frame we can see the chloroform phase containing $\mathrm{PS}_{16 \mathrm{k}}-\mathrm{PEO}_{7.5 \mathrm{k}}$ and the water phase being deposited from the top. In the second frame, 5 seconds after the formation of the chloroform-water interface the spontaneous formation of droplets of water in chloroform is clearly visible. 


\section{Scanning Electron Microscopy Images of the Aggregates}

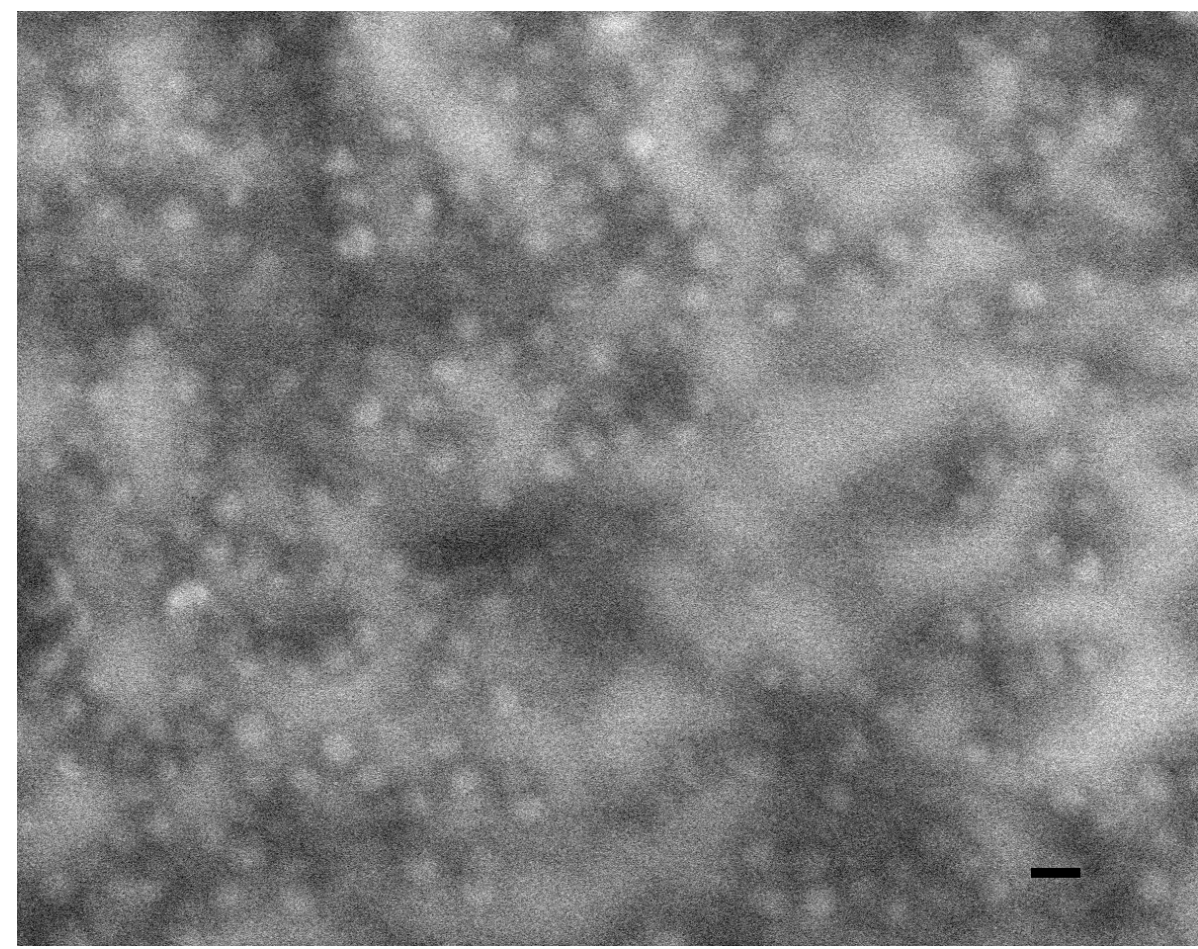

Figure S2. Spherical micelles formed without evaporation from a planar interface between chloroform containing $\mathrm{PS}_{9.5 \mathrm{k}}-\mathrm{PEO}_{18 \mathrm{k}}$ and water: $5 \mu \mathrm{L}$ of the upper clear water phase were dried on a silicon wafer and imaged by SEM. Upon drying the chloroform in water droplets transform into spherical micelles with diameters about $55 \mathrm{~nm}$. Scale bar $100 \mathrm{~nm}$.

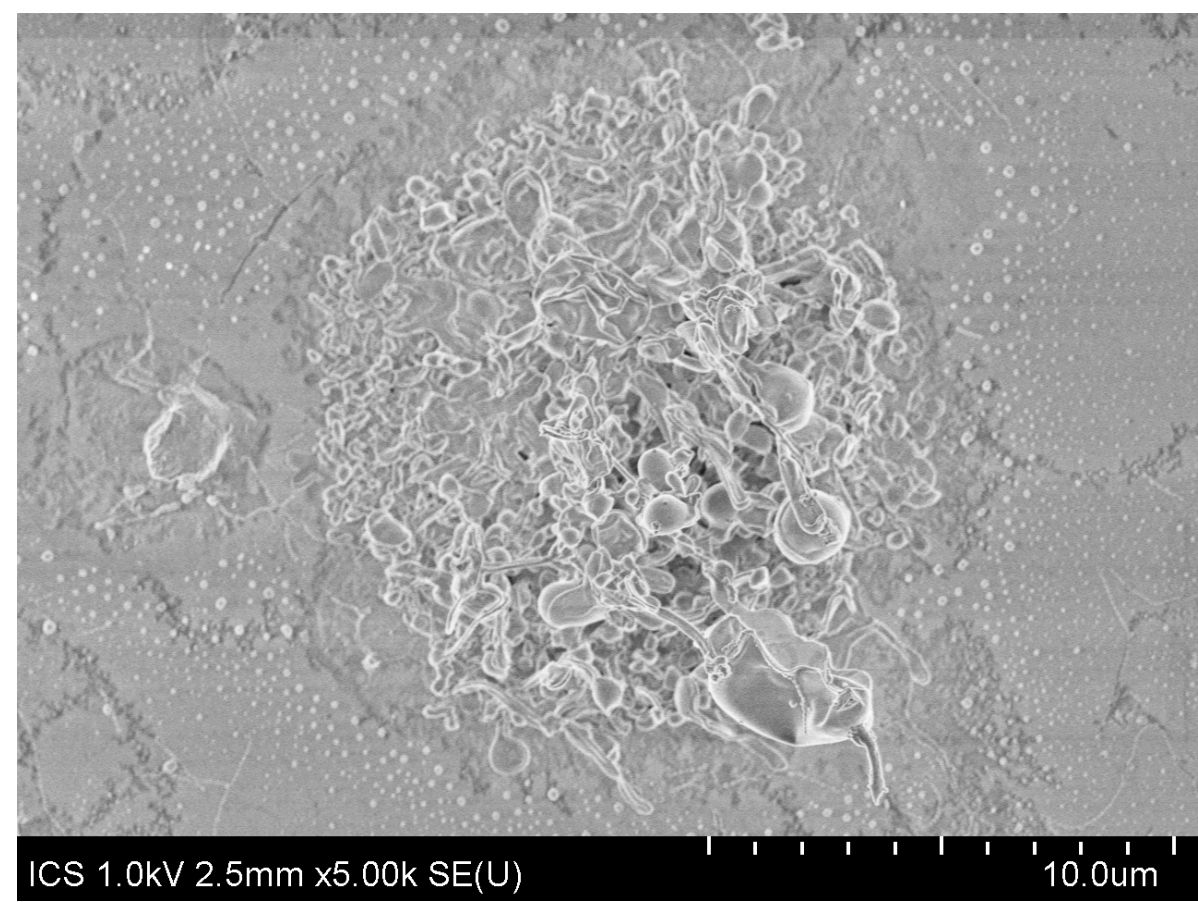

Figure S3. Budding vesicle formed from an adsorbed droplet of chloroform containing $\mathrm{PS}_{16 \mathrm{k}}-\mathrm{PEO}_{7.5 \mathrm{k}}$. The adsorbed droplets form a budding vesicle and a series of smaller spherical vesicles and elongated 
strings of vesicles. The former can be seen surrounding the larger aggregate while the latter are shown in more detail in Figure S4.

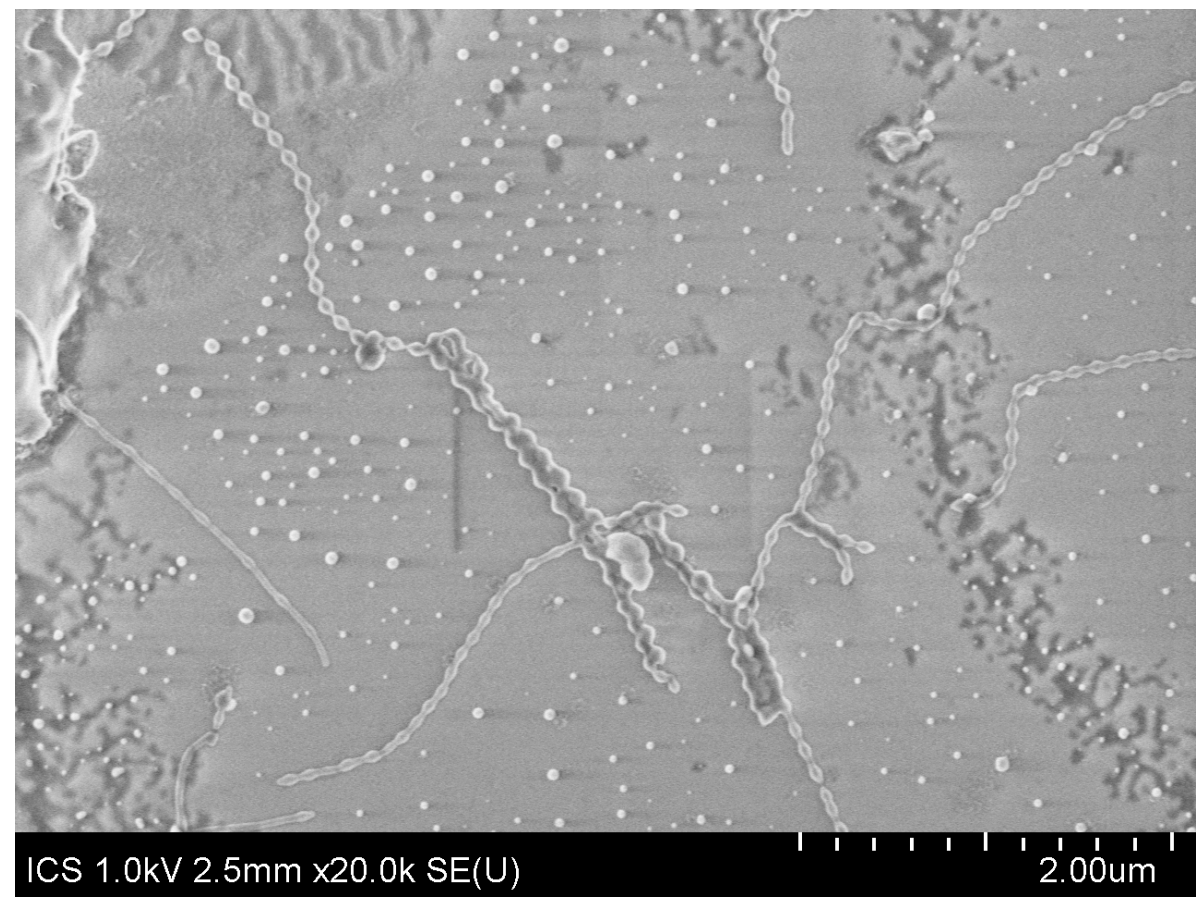

Figure S4. Detail of the strings of vesicles surrounding the budding vesicle formed from adsorbed droplets containing $\mathrm{PS}_{16 \mathrm{k}}-\mathrm{PEO}_{7.5 \mathrm{k}}$.

\section{Laser Scanning Confocal Microscopy}

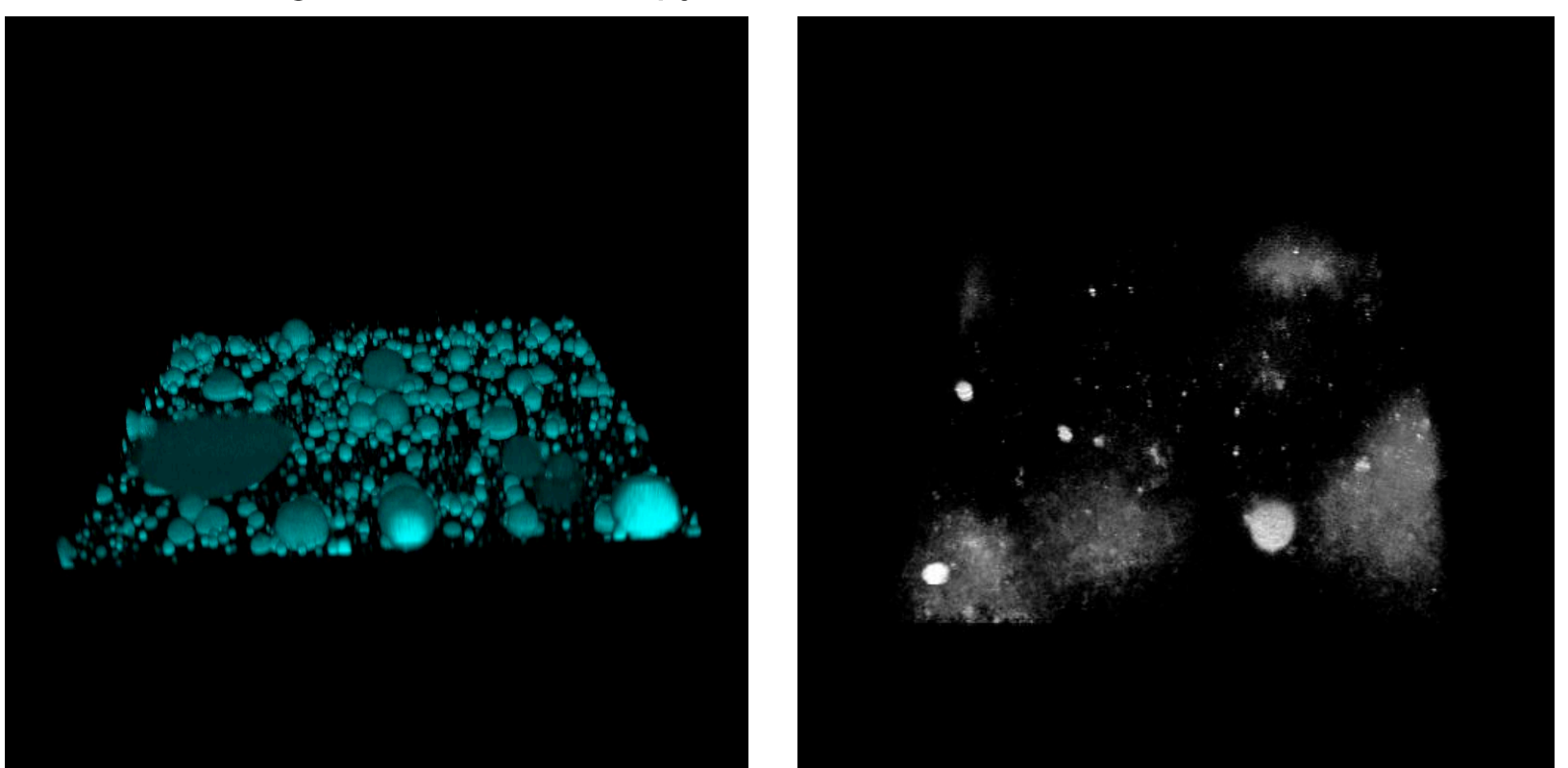

Figure S5. (left) LSCM of chloroform/PS ${ }_{16 \mathrm{k}^{-}}-\mathrm{PEO}_{7.5 \mathrm{k}^{-}}$droplets at the bottom of the imaging chamber (right) View of the same sample after the formation of micelles. Some droplets produced clearly visible 
elongated micelles while most gave spherical micelles too small for optical microscopy. The chlorofom contains a small amount of Dil dye. See also the .avi files showing the data from different viewpoints.

\section{List of videos}

Seven videos are available on the publisher's website illustrating various aspects of our experiences:

Movie 1) Spontaneous_emulsification_planar_interface;

Violent penetration of upper water phase into the bottom chloroform phase containing $100 \mathrm{mg} / \mathrm{mL} \mathrm{PS}{ }_{16 \mathrm{k}}-\mathrm{PEO}_{7.5 \mathrm{k}}$.

Movie 2) Floating-droplets_ps16k-peo7_5k_elongated_formation:

Elongated micelles are formed from floating emulsion droplets containing $10 \mathrm{mg} / \mathrm{mL} \mathrm{PS}_{16 \mathrm{k}}-\mathrm{PEO}_{7.5 \mathrm{k}}$. The elongated micelles are clearly visible thanks to the fluorescent dye Dil.

Movie 3) Floating-droplets_ps9_5k-peo18k_spherical_formation:

Spherical micelles are formed from floating emulsion droplets containing 10 $\mathrm{mg} / \mathrm{mL} \mathrm{PS}_{9.5 \mathrm{k}}-\mathrm{PEO}_{18 \mathrm{k}}$. The droplets shrink in size until they disappear.

Movie 4) Adsorbed_droplets_ps16k-peo7_5k_budding-vesicles_formation:

Vesicles and budding vesicles are formed from adsorbed emulsion droplets containing $10 \mathrm{mg} / \mathrm{mL} \mathrm{PS}_{16 \mathrm{k}}-\mathrm{PEO}_{7.5 \mathrm{k}}$.

Movie 5) Adsorbed-droplets_ps9_5k-peo18k_elongated_formation:

Elongated micelles are formed from adsorbed emulsion droplets containing $10 \mathrm{mg} / \mathrm{mL} \mathrm{PS}_{9.5 \mathrm{k}}-\mathrm{PEO}_{18 \mathrm{k}}$.

Movie 6) LSCM_before

Figure S5 left, from different viewpoints

Movie 7) LSCM_after

Figure S5 right, from different viewpoints 